\title{
Effects of Prime Task on Affective Priming By Facial Expressions of Emotion
}

\author{
Luis Aguado, Ana Garcia-Gutierrez, Ester Castañeda, and Cristina Saugar \\ Universidad Complutense
}

\begin{abstract}
Priming of affective word evaluation by pictures of faces showing positive and negative emotional expressions was investigated in two experiments that used a double task procedure where participants were asked to respond to the prime or to the target on different trials. The experiments varied between-subjects the prime task assignment and the prime-target interval (SOA, stimulus onset asynchrony). Significant congruency effects (that is, faster word evaluation when prime and target had the same valence than when they were of opposite valence) were observed in both experiments. When the prime task oriented the subjects to an affectively irrelevant property of the faces (their gender), priming was observed at SOA $300 \mathrm{~ms}$ but not at SOA $1000 \mathrm{~ms}$ (Experiment 1). However, when the prime task assignment explicitly oriented the subjects to the valence of the face, priming was observed at both SOA durations (Experiment 2). These results show, first, that affective priming by pictures of facial emotion can be obtained even when the subject has an explicit goal to process a non-affective property of the prime. Second, sensitivity of the priming effect to SOA duration seems to depend on whether it is mediated by intentional or unintentional activation of the valence of the face prime.
\end{abstract}

Keywords: affective priming, facial expressions of emotion, automatic and controlled processing

En dos experimentos con un procedimiento de doble tarea en el que los participantes habían de responder al "prime" o al "target" en diferentes ensayos, se estudió el efecto de "priming" sobre la evaluación afectiva de palabras, producido por imágenes de caras que mostraban expresiones emocionales positivas o negativas. En los experimentos se varió entre sujetos la naturaleza de la tarea a realizar respecto al "prime" y la duración del intervalo prime-target (SOA). En ambos experimentos se obtuvieron efectos significativos de la congruencia, es decir, evaluación más rápida de las palabras cuando "prime" y "target" eran de la misma valencia que cuando eran de valencia opuesta. Cuando la tarea con el "prime" orientaba a los sujetos hacia una propiedad afectivamente irrelevante de las caras (su género), se obtuvo "priming" con SOA de $300 \mathrm{mseg}$, pero no con SOA de $1000 \mathrm{mseg}$ (Experimento 1). En cambio, cuando la tarea con el "prime" orientaba expresamente a los sujetos hacia la valencia de la cara, se obtuvo "priming" con ambas duraciones de SOA. Estos resultados muestran, en primer lugar, que puede obtenerse "priming" afectivo con imágenes de expresiones faciales de la emoción aun cuando el sujeto esté expresamente orientado a procesar una propiedad no afectiva del "prime". En segundo lugar, la sensibilidad del "priming" a la duración del SOA parece depender de si el efecto está mediado por la activación intencional o no intencional de la valencia de la cara que actúa como "prime".

Palabras clave: "priming" afectivo, expresiones faciales de la emoción, procesamiento automático y controlado

This work was supported by Project BSO2003-03661, from the Spanish Ministerio de Ciencia y Tecnología.

Correspondence should be addressed to Luis Aguado, Facultad de Psicología, Campus de Somosaguas, 28023 Madrid, Spain. Phone number: 91 3943161. FAX: 91 3943189. E-mail: laguado@psi.ucm.es 
A widely accepted view is that valence of an affective stimulus is activated automatically and unintentionally when the stimulus is perceived. However, the precise nature of this activation process and its specific consequences are a matter of debate. One hypothesis is that the process of valence activation takes the form of an affective evaluation or cognitive evaluative response automatically triggered by incoming stimuli. According to this hypothesis, stimuli are automatically evaluated as "positive" or "negative," "good" or "bad," "pleasant" or "unpleasant," without the need of deliberation or intention to evaluate (Bargh, Chaiken, Govender, \& Pratto, 1992; Fazio, 2001). An experimental paradigm frequently used to study automatic activation of stimulus valence is a variation of semantic priming called affective priming. In the standard procedure, the subject has to evaluate the valence of a target word (e.g., as "pleasant" or "unpleasant"). Primes and targets are stimuli with positive or negative valence and the critical comparison is between congruent trials, where prime and target are of the same valence, and incongruent trials, where the stimuli are of opposed valence. The affective priming effect is typically defined as a faster reaction time (RT) to the target on congruent than on incongruent trials (that is, an affective congruency effect, e.g., Fazio, Sanbonmatsu, Powell, \& Kardes, 1986; for reviews see Fazio, 2001, and Klauer \& Musch, 2003). This effect has been shown with a variety of stimuli, such as words and images (Spruyt, Hermans, De Houwer, \& Eelen, 2002), facial expressions of emotion (Stenberg, Wiking, \& Dahl, 1998) sounds (Sollberger, Reber, \& Eckstein, 2003), and smells (Hermans, Baeyens, \& Eelen, 1998).

Affective priming effects have been typically obtained with short prime-target onset time intervals. A typical finding is priming at $300 \mathrm{~ms}$ but not at $1000 \mathrm{~ms}$ stimulus onset asynchrony (SOA)(e.g., Fazio et al., 1986; Hermans, Spruyt, \& Eelen, 2003), though some studies have found significant priming effects only at shorter SOAs. For example, Hermans, De Houwer, and Eelen (2001) carried out a within-subjects analysis of SOA duration, using five durations ranging from -150 to $450 \mathrm{~ms}$, with a word-word paradigm and an affective evaluation task. Significant priming effects were obtained only at 0 and $150 \mathrm{~ms}$. Traditionally, priming effects observed at short, but not at long, SOAs have been taken as evidence of automaticity (Neely, 1977; Posner \& Snyder, 1975), based on the assumption that automatic processes are unintentional, occur at early stages of processing, and cannot be deliberately counteracted at such short intervals. Coupled with evidence of affective congruency effects with subliminally presented primes (Hermans, Spruyt, De Houwer, Eelen, 2003), this sensitivity to SOA duration seems to suggest that affective priming effects are a consequence of the automatic and unintentional activation of affective valence. In fact, affective priming is obtained even though in most studies the participant is explicitly discouraged from paying attention to the prime (e.g., Hermans, Spruyt, \&
Eelen, 2003). However, an open issue is whether affective priming can still be obtained when the participant is explicitly instructed to process an affectively irrelevant property of the prime. Priming observed under these conditions would further reinforce the idea that affective evaluation can be activated independently from the explicit processing goals of the subject.

In the experiments here reported, we used a novel primetarget, double-task procedure, with pictures of facial expressions of emotion as primes and affectively positive and negative words as targets. We used this procedure with the aim of evaluating the effects of different prime tasks on affective priming. Pictures of facial expressions of emotion (happy, angry, and fearful faces) were used because they are well suited for this task. First, facial expressions of emotion are naturalistic affective or emotional stimuli that share some properties with other types of affective stimuli. For example, expressive facial muscles have been shown to respond in a different manner to images of positive and negative emotional faces in studies measuring electromyographic (EMG) activity in the perceiver (e.g., Dimberg \& Karlsson, 1997). The short latency with which differential EMG reactions are evoked (around 400-500 ms) and the fact that this activity is observed even under backward masking conditions (Dimberg, Thunberg, \& Elmehed, 2000) suggest that the valence of facial expressions is quickly and automatically decoded without the need of deliberate evaluation. A second suitable property of facial expressions of emotion is that they can be categorized in several alternative ways, so the participant can be oriented to explicitly pay attention to affectively relevant (valence) or irrelevant (gender) properties of the prime.

In the experiments here reported, the participants were always asked to evaluate a target word. However, whereas in Experiment 1 they were asked to identify the gender of the face prime (an affectively irrelevant task), in Experiment 2 , the participants had to evaluate affectively both the prime and the target. The main goal of this double-task procedure was to promote different modes of processing the prime, only one of which required explicit attention to the affective meaning of the face primes. The procedure included two types of trials: the usual prime-target trials, where the target had to be responded to, and prime-only trials, where a response to the prime was required. Prime-target trials and prime-only trials appeared unpredictably and in the same proportion, so that on a specific trial, the participant could not know in advance whether the prime or the target had to be responded to. In this way, we tried to orient the participant to an affectively relevant (valence) or irrelevant (gender) feature of the prime. As the prime is explicitly evaluated only in the valence-valence task, and as the participant is oriented to an affectively irrelevant property of the face in the gender-valence task, the comparison between both tasks allows in principle an evaluation of the effects of unintentional activation of the affective valence 
of the prime (in the gender-valence condition) with those of intentional or strategic activation of valence (in the valence-valence condition).

In both experiments, two SOA durations were used (300 and $1000 \mathrm{~ms}$ ). Our prediction was that priming with a short SOA duration (300 ms) should be observed both in the gender-valence condition (Experiment 1) and in the valencevalence condition (Experiment 2). However, we also predicted that priming at a long SOA duration (1000 ms) would be observed only in the valence-valence condition, when the participants were explicitly asked to evaluate the primes. This second prediction is based on the fact that the effects of automatic or unintentional activation of affective evaluation observed in affective priming experiments is short-lived, whereas valence activation under explicit instructions to evaluate can be maintained as long as it is required to meet the goals of the task.

\section{Experiment 1}

In Experiment 1, a double gender-valence task was used. The double-task procedure included two different types of trials. In prime-only trials, the participants had to identify the gender of the face prime, whereas in prime-target trials, the participant had to evaluate the target word. An identical proportion of both trials, randomly distributed, was included. Given the unpredictability of the specific task to perform on a specific trial, the participants were forced to attend to the gender of the faces on every trial. Priming with the gender-valence task would go beyond the usual demonstration that the effect is obtained without the need of an explicit goal to evaluate. It would demonstrate, furthermore, that this occurs even when the subject is asked to process an affectively irrelevant property of the prime.

In order to test the sensitivity of affective priming with the gender-valence task to SOA duration, we carried out a between-subjects comparison of two durations, 300 and 1000 ms, with affective face-word congruency as a within-subjects factor. Our specific prediction was that, as long as, under the specific conditions of this double task, affective priming depends on automatic or unintentional activation of prime valence, significant priming should be manifest only at the shorter, $300 \mathrm{~ms}$ SOA.

\section{Method}

\section{Participants}

Participants were 39 psychology students (20 in the SOA 300 condition and 19 in the SOA 1000 condition) who participated in the experiments for course credit. There were 22 females and 17 males, with ages 17-28 years (mean 21.18).

\section{Apparatus and Stimuli}

Presentation of stimuli and registration of responses was controlled through the software E-Prime 1.1. The program was run on a Pentium III computer with a $64 \mathrm{Mb}$ RAM memory and the stimuli were presented on a VGA 17 " monitor (refresh rate $60 \mathrm{~Hz}$ ). Subjects were seated at a distance of $50 \mathrm{~cm}$ from the screen. Responses were registered through a computer keyboard during the familiarization and the face evaluation phases and through a five-key response box (PST Serial Response Box, 200A) during the priming phase. Sessions were carried out individually in a soundproof, dimly lighted room.

The stimuli used as primes were 16 black and white pictures from the Ekman and Friesen (1975) collection. Of these, 8 were of women and 8 of men. Two pictures of each model were included, one showing a happy expression and the other one showing a fearful or an angry expression. Primes of positive valence were 8 pictures of smiling faces. Six of these pictures showed open-mouth smiles and two closed-mouth smiles (one female, one male). Primes of negative valence were 4 pictures of faces showing a fearful expression and 4 faces showing an angry expression (two of them showing an open mouth and two showing a closed mouth). Male and female models were equally represented in each emotional category. During the practice phase, 8 additional faces from the Ekman and Friesen collection were used, all showing a neutral expression and taken from different models from those that would be used on the experimental phase. Pictures $(11.29 \times 16.95 \mathrm{~cm})$ were cropped to conceal most of the hair. Stimuli were presented on the center of the screen on a black background. The faces subtended a horizontal visual angle of $9.09^{\circ}$ and a vertical angle of $14.03^{\circ}$.

Target stimuli were 64 Spanish nouns with positive or negative valence (32 positive, 32 negative), selected according to the pleasantness dimension (from 1maximum negative-to 7-maximum positive) and the imaginability, syllable number, and word length ratings of the normative study of Algarabel (1996). Mean pleasantness rating for the selected positive and negative words was 5.85 and 1.78 , respectively, $t(1,62)=72.62$, $p=.000$. Positive and negative words were equated in frequency of use, imaginability, number of syllables, and word length. Mean frequency, according to the Spanish norms of Alameda and Cuetos (1995), was 47.00 for positive and 37.12 for negative words, $t(1,62)=1.26, p$ $>$.05. Mean imaginability was 5.04 for positive and 4.8 for negative words, $t(1,62)=1.043, p>.05$. Mean syllable number was 3.09 for positive and 2.93 for negative words, $t(1,62)=1.03, p>.05$. Lastly, word length was 7.37 for positive and 6.93 for negative words, $t(1,62)=1.09, p>.05$. Words were presented on the center of the screen, written in white letters (Arial 18 font) on a blue background 


\section{Procedure}

Instructions and familiarization. The instructions, presented self-paced on the computer screen, described the task to be performed and stressed that responses should be fast (see Appendix 1). After the instructions, the familiarization phase begun. This phase was included with the aim of familiarizing the subjects with the pictures that would later be used as primes. On the 16 familiarization trials (one per face) the subjects had to identify the gender of the faces, pressing $\mathrm{H}$ for male ("hombre" in Spanish) and $\mathrm{M}$ for female ("mujer" in Spanish) on the computer keyboard.

Priming phase. A priming procedure with faces as primes and words as targets was employed, with prime-target affective congruency being the within-subjects factor. The priming phase included two types of trials, prime-target trials and prime-only trials. This phase started with a practice block of 32 trials (16 prime-target and 16 prime-only trials). The primes of the practice trials were neutral faces and the targets positive and negative words that would not be used in the experimental phase. Responses from these trials were not considered for statistical analysis.

The experimental priming phase comprised 128 trials, divided in two blocks of 64 trials. In each block, there were 32 prime-target and 32 prime-only trials. All trials started with the presentation of an asterisk that acted as a fixation point, appearing on the center of the screen, with a duration of $500 \mathrm{~ms}$. Immediately after this, the face prime was presented with a duration of $250 \mathrm{~ms}$. On prime-target trials, the prime and the target were separated by an SOA of 300 or $1000 \mathrm{~ms}$, depending on the group. The target word remained on the screen until the subject responded or until a maximum of $2000 \mathrm{~ms}$ had elapsed. On these trials, the task of the subjects was to evaluate the words as "pleasant" or "unpleasant." Half of the trials in each block were congruent, with the face and the word sharing the same affective valence (e.g., happy face $\rightarrow$ "aurora [sunrise]"), and half incongruent, where the face and the word were of opposite valence (e.g., happy face $\rightarrow$ "cárcel [jail]"). Depending on the combination of prime and target valences, there were two types of congruent trials (positive-positive and negative-negative) and two types of incongruent trials (positive-negative and negative-positive). The specific primetarget pairings appearing on each trial were determined randomly for each subject in order to prevent any potential influence of particular face-word pairings.

On prime-only trials, the target was substituted for a question mark appearing on the center of the screen at the same time that the target should have appeared. The subjects were instructed that on the trials where a question mark appeared after the prime, they should report the gender of the face. Half of these trials had positive and half negative faces, each with the same proportion of male and female faces. The same set of faces was employed in prime-target and prime-only trials. The two types of trials were randomly distributed over the priming phase, so that the specific task to be performed on a specific trial was unpredictable until the target or the prompt to report the gender of the face appeared. Both tasks, target evaluation and prime gender identification, were performed on the response box, with different keys being assigned to each task (1 and 2 or 4 and 5 , counterbalanced). Duration of SOA was varied between groups, with one group having an SOA of $300 \mathrm{~ms}$ and the other group an SOA of $1000 \mathrm{~ms}$.

Face evaluation phase. After the priming phase, all the faces employed as primes on the experimental priming phase were presented again in random order. The participants were instructed to evaluate each face as to its pleasantness on a continuous 9-point scale, with 1 being very unpleasant and 9 very pleasant. Responses were entered by pressing keys 1 to 9 on the computer keyboard. This phase was included with the aim of having an explicit and graded measure of the affective evaluation of the faces by the participants.

\section{Results and Discussion}

In this and the following experiment, only reaction times from correct trials were considered for the main analysis. Moreover, outliers (less than $200 \mathrm{~ms}$ or more than $2000 \mathrm{~ms}$ RT) were excluded from analysis (1.2\% of total data, in the present experiment). Mean target accuracy did not differ significantly between congruent (.94) and incongruent (.95) trials $(p>.05)$. Figure 1 shows mean RT on congruent and incongruent trials for the two SOA conditions. Mean incongruent-congruent difference was $32.5 \mathrm{~ms}$ for group 300 and $-6.8 \mathrm{~ms}$ for group 1000. A Group $\times$ Congruency ANOVA, with Group as a between subjects factors and Congruency as the within-subjects variable, was performed on RT data. There were no significant effects of Congruency, $F(1,37)=1.78, p>.05$ or Group $(F<1)$. However, the most important aspect of the results was a significant Congruency $\times$ Group interaction, $F(1,37)=4.17, p<.05$.

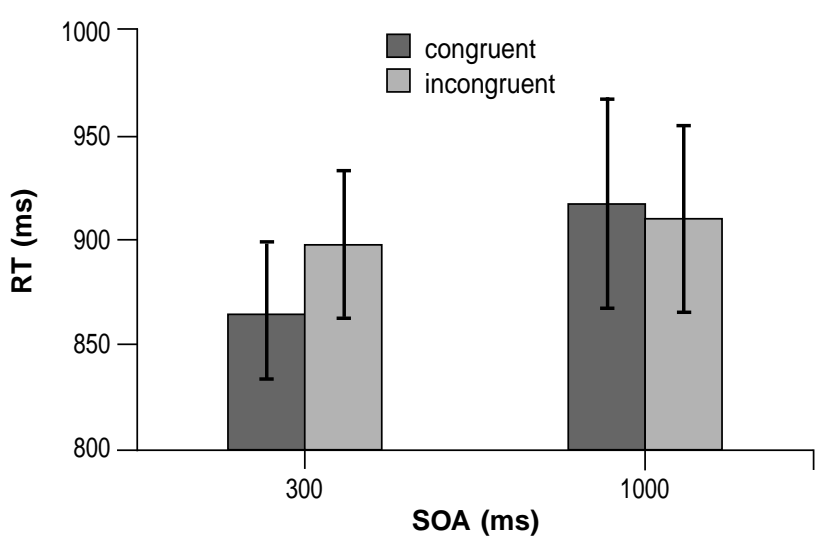

Figure 1. Experiment 1: Effect of prime-target affective congruency as a function of SOA duration (bars represent SE). 
This interaction was further explored by means of post-hoc comparisons with the Bonferroni adjustment. These comparisons revealed a significant difference between congruent and incongruent trials only in group 300.

Mean RTs for positive and negative targets collapsed over the two groups were $889(S D=188.23)$ and $899 \mathrm{~ms}(S D=$ 171.27), respectively. The difference between these two conditions was not significant, $t(38)=.75$. Mean response accuracy on prime gender identification trials for the two groups collapsed was .94, with a mean RT of $605 \mathrm{~ms}$ on trials with correct responses. Lastly, mean evaluations during the evaluation phase were $6.93(S D=1.15)$ for positive and $3.18(S D=1.16)$ for negative faces, $t(38)=11.11, p<.05$.

In this experiment, a significant affective priming effect was observed with a double prime/target task where the subjects were unpredictably asked to identify the gender of a face prime or to evaluate affectively a target word. The fact that significant priming was obtained in spite of an explicit goal to attend to an affectively irrelevant property of the face primes suggests that the source of the effect was the unintentional, automatic activation of affective valence by pictures of facial expressions of emotion. This interpretation is further supported by the sensitivity of the affective priming effect to SOA duration, with priming observed only at $300 \mathrm{~ms}$ SOA, but not at a longer, $1000 \mathrm{~ms}$ SOA. This sensitivity to SOA duration is coincident with the results usually obtained in affective priming studies, where priming is not observed at SOA durations longer than 300-400 ms (e.g., Fazio et al., 1986; Hermans et al., 2001).

Given that these results were obtained with a double prime/target task that oriented the subjects to an affectively irrelevant property of the prime, it would be interesting to see what the effect would be of SOA duration when subjects are given a prime task assignment that requires explicit processing of stimulus valence. Should an affective congruency effect be obtained under these conditions, it might be reasonably attributed to the explicit goal to evaluate the primes. And if this were so, it can be predicted that priming should be observed both at short and long SOA durations. Consequently, in Experiment 2 a double prime/target task similar to that employed in Experiment 1 was used, though in this case, subjects were explicitly instructed to evaluate the face primes.

\section{Experiment 2}

In Experiment 1, priming of evaluative responses by facial expressions of emotion was observed although subjects were assigned an affectively irrelevant prime task. In the following experiment, a similar unpredictable double task procedure was employed. However, this time, the subjects were asked to evaluate affectively both the prime and the target. This assignment was given in order to promote a strategic set to attend to the affective meaning of the faces.
Our prediction was that this procedure should have a strong effect on evaluative responses to the targets and that a clear difference in response speed between congruent and incongruent trials should be observed. However, as this effect would be based on strategic_and intentional processes that can be voluntarily maintained to meet the requirements of the task, we also expected that it would be observed at both short and long SOA durations. As the subjects could not know what specific task they should perform on a given trial until the target or the prompt to evaluate the prime appeared, they would be forced to evaluate the primes on all trials. Thus, on prime-target trials, the evaluative response to the prime would still be activated when the target appeared and this would happen at both SOA durations.

\section{Method}

\section{Participants}

Participants were 39 psychology students. There were 34 women and five men, with ages of 18-22 years (mean 18.9).

\section{Procedure}

Apparatus and materials were the same as in the previous experiment. The procedure was also identical to that of Experiment 1 and had the same familiarization, practice, priming, and evaluation phases. The priming phase included prime-target and prime-only trials, and primes and targets were presented with the same parameters as in Experiment 1. The only variation concerned the prime task assigned to the subjects. In the present experiment, the participants were instructed that on the trials where a question mark appeared after the prime, they should report the affective valence of the face ("pleasant" or "unpleasant"). Half of these trials had positive and half negative faces. The same face stimuli were employed in both types of trials. The inclusion of prime-only trials resulted in a total of 32 trials in the practice block and 128 trials in the experimental priming phase. The two types of trials were randomly distributed. Both prime and target evaluation responses were performed using keys 1 and 5 from the response box, the specific assignment being counterbalanced across subjects. SOA duration was varied between groups, with one group having an SOA of $300 \mathrm{~ms}$ and the other group an SOA of $1000 \mathrm{~ms}$.

\section{Results and Discussion}

RTs falling outside the predetermined maximum and minimum cut-off points were excluded from analysis $(0.84 \%$ of total data). Table 1 shows error rates of responses to the target, corresponding to the priming phase of Experiment 2. A Congruency $\times$ Group ANOVA performed on target 
accuracy data gave a significant effect of Congruency, $F(1,37)$ $=7.07, p=<.05$, but not of Group or the interaction $(F \mathrm{~s}<$ 1). Figure 2 shows the main results of the priming phase of Experiment 2 in terms of RT. It can be seen that evaluative responses to the target were faster on congruent than on incongruent trials at both SOA durations. The incongruentcongruent difference was $83 \mathrm{~ms}$ in group 300 and $44 \mathrm{~ms}$ in group 1000. A Group $\times$ Congruency ANOVA, with repeated measures on the second variable, gave a significant effect of congruency, $F(1,37)=6.43, p<.05$, but not of group or the interaction $\left(F_{\mathrm{S}}<1\right)$. Thus, in can be concluded that in Experiment 2 a strong congruency effect was obtained and that this effect was maintained over the two SOA durations. Moreover, in contrast with the results of Experiment 1, this effect was manifested as significant differences in both error rates and reaction times to the target. Both measures, error rate and $\mathrm{RT}$, were consistent in the sense that a higher error rate was observed in the incongruent condition, which also produced longer evaluation times.

Mean RTs for positive and negative targets collapsed over the two groups were $827.58(S D=131)$ and $803 \mathrm{~ms}$ $(S D=149)$, respectively. The difference between these two conditions was not significant, $t(1,38)=1.86, p<.05$. Response accuracy and RT were also recorded on prime evaluation trials. Mean prime accuracy for the two groups collapsed was .94 . On prime evaluation trials with correct responses, mean RT was 629 ms. Lastly, mean evaluation responses during the face evaluation phase were 6.82 (SD $=1.52)$ for positive and $3.21(S D=1.82)$ for negative faces, $t(1,38)=8.33, p<.05$.

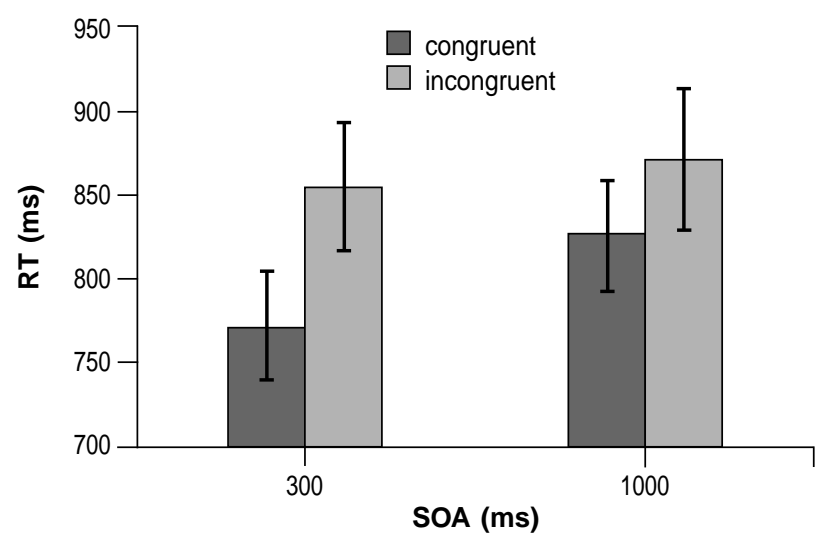

Figure 2. Experiment 2: Effect of prime-target affective congruency as a function of SOA duration (bars represent SE).
The results of this experiment showed a strong congruency effect on word evaluation responses, produced by affectively congruent or incongruent picture primes of happy, angry, or fearful facial expressions. In contrast with the results of Experiment 1, this effect was maintained when SOA duration was increased from 300 to $1000 \mathrm{~ms}$ and was manifested both in RTs and in error rates. This discrepancy was surely due to the only procedural variation between Experiments 1 and 2, that is, the different prime task assignment. Whereas in Experiment 1, a gender identification task was assigned, in the present experiment the subjects were instructed to evaluate affectively the primes. As the subjects were asked to explicitly judge the valence of the faces, the evaluation task obviously required intentional activation of affective valence. And as the task to be performed on a specific trial was unpredictable until the end of the prime, where the target or the question mark appeared, a plausible assumption is that the evaluative response to the prime was deliberately activated on all trials. In this way, when the target appeared on prime-target trials, the speed of word evaluation depended on the congruency or incongruency between prime and target valence. And as prime evaluation was in this case controlled_and intentional, it was maintained over the SOA duration and modulated affective evaluation of the target even at the $1000 \mathrm{~ms}$ duration.

\section{General Discussion}

The two experiments here reported explored the affective priming, or affective congruency effect, with pictures of facial expressions of emotions as primes and affectively positive or negative words as targets. In both experiments, the participants had to evaluate the valence of the target. The affective priming effect, consisting of shorter reaction times on affectively congruent than on affectively incongruent trials, was shown to be modulated by the specific mode of processing the prime induced by two different prime tasks. In Experiment 1, affective priming by emotional faces was observed with a double gender-valence task, where the subjects were unpredictably asked to identify the gender of the faces or to evaluate the valence of the target. This effect was observed on target evaluation trials at an SOA duration of $300 \mathrm{~ms}$, but disappeared when this duration was increased to $1000 \mathrm{~ms}$. In Experiment 2, priming was observed with an unpredictable double valence-valence

Table 1

Experiment 2: Mean Error Rate on Congruent and Incongruent Trials

\begin{tabular}{lccc}
\hline SOA & Congruent & Incongruent & Difference (Incongruent-Congruent) \\
\hline 300 & $3.47 \%$ & $11.5 \%$ & 8.03 \\
1000 & $4.3 \%$ & $11.5 \%$ & 7.2 \\
\hline
\end{tabular}


task, where the prime or the target had to be evaluated affectively. In this experiment, priming was observed at both SOA durations (300 and $1000 \mathrm{~ms}$ ).

The results of Experiment 1 show that affective priming can be obtained not only when the participants have no explicit goal to evaluate but even when they are explicitly instructed to attend to an affectively irrelevant property of the prime. This, together with the sensitivity of the priming effect to SOA duration, is consistent with an interpretation in terms of unintentional or automatic activation of prime valence. The present results are thus consistent with those obtained with the affective version of the Simon task (De Houwer \& Eelen, 1998), which also shows automatic processing of valence in the presence of a goal to process a non-affective property of the stimulus. A plausible interpretation of the fact that priming was obtained at both SOA durations in Experiment 2 is that, given that the participants had an explicit goal to evaluate the faces, valence of the primes was activated through a strategic and intentional process.

It is interesting to consider the results of Experiment 1 in relation to those from studies on prime task effects in semantic priming. A number of studies have found that prime task is a strong modulator of word-word semantic priming. A usual finding is that tasks that orient the subject to superficial, low-level properties of the primes (e.g., letter identification) reduce or eliminate semantic priming, whereas tasks that involve semantic activation (naming or lexical decision) promote it (Henik, Friedrich, \& Kellogg, 1983; see Maxfield, 1997, for a review). Prime task effects have received much attention due to their implications for the debate on automaticity of semantic activation. Specifically, it has been stated that modulation by prime task is difficult to reconcile with models of semantic priming relying only on automatic, nonstrategic mechanisms (Stolz \& Besner, 1999). In contrast with the usual results of prime task experiments with semantic priming, we obtained an affective priming effect in spite of assigning the participants a prime task that was irrelevant with regard to affective evaluation. An interpretation of this discrepancy is that whereas semantic processing is subjected to controlled processes in the case of words, being impaired by tasks that orient the subjects away from semantic processing, valence activation of an affective picture is automatic and can run in parallel with the processing of nonaffective properties of the stimulus. Glaser and Glaser (1989), for example, have proposed that whereas words have privileged access to the lexical system, pictures have a privileged, not mediated access, to the semantic system. Results consistent with this proposal have been presented by De Houwer and Hermans (1994), who showed that affectively incongruent word distracters did not influence affective categorization of pictures in a Stroop paradigm. However, these authors showed that affectively incongruent picture distracters did influence affective categorization of words. Assuming that affective information is stored in the semantic system, De Houwer and Hermans interpreted their results as showing that this system is accessed through a direct route by visual stimuli.

In conclusion, the present series of experiments has shown, first, that affective priming with pictures of facial expressions of emotion can be obtained when the participant is instructed to attend to an affectively irrelevant property of the prime. Second, sensitivity to SOA duration depends on whether priming is based on intentional or unintentional activation of prime valence. When priming is based on unintentional or automatic processes, it is observed only at short SOA intervals. However, it can be observed at both short and long intervals when it is based on intentional activation of valence.

\section{References}

Algarabel, S. (1996). Indices de interés lingüístico de 1917 palabras castellanas. Cognitiva, 8, 43-88.

Alameda, J.R., \& Cuetos, F. (1995). Diccionario de frecuencias de las unidades lingüísticas del castellano (Vol.1). Oviedo, Spain: Servicio de Publicaciones de la Universidad de Oviedo.

Bargh, J., Chaiken, S., Govender, R., \& Pratto, F. (1992). The generality of the automatic attitude activation effect. Journal of Personality and Social Psychology, 62, 893-912.

De Houwer, J., \& Eelen, P. (1998). An affective variant of the Simon paradigm. Cognition and Emotion, 12, 45-61.

De Houwer, J., \& Hermans, D. (1994). Differences in the affective processing of words and pictures. Cognition and Emotion, 8 , $1-20$.

Dimberg, U., \& Karlsson, B. (1997). Facial reactions to different emotionally relevant stimuli. Scandinavian Journal of Psychology. 38, 297-303.

Dimberg, U., Thunberg, M., \& Elmehed, K. (2000). Unconscious facial reactions to emotional facial expressions. Psychological Science, 11, 1, 86-89.

Ekman, P., \& Friesen, W. (1975). Pictures of facial affect. Palo Alto, CA: Consulting Psychologists Press.

Fazio, R. (2001). On the automatic activation of associated evaluations: An overview. Cognition and Emotion, 15, 115-141.

Fazio, R.H., Sanbonmatsu, D.M., Powell, M.C., \& Kardes, F.R. (1986). On the automatic activation of attitudes. Journal of Personality and Social Psychology, 50, 229-238.

Henik, A., Friedrich, F.J., \& Kellogg, W.A. (1983). The dependence of semantic relatedness effects upon prime processing. Memory \& Cognition, 11, 366-373.

Hermans, D., Baeyens, F., \& Eelen, P. (1998). Odours as affectiveprocessing context for word evaluation: A case of cross-modal affective priming. Cognition and Emotion. 12, 601-613.

Hermans, D., De Houwer, J., \& Eelen, P. (2001). A time course analysis of the affective priming effect. Cognition and Emotion, 15, 143-165.

Hermans, D., Spruyt, A., De Houwer, J., \& Eelen, P. (2003). Affective priming with subliminally presented pictures. Canadian Journal of Experimental Psychology. 57, 97-114. 
Hermans, D., Spruyt, A., \& Eelen, P. (2003). Automatic affective priming of recently acquired stimulus valence: Priming at SOA 300 but not at SOA 1000. Cognition and Emotion, 17, 83-99.

Klauer, K., \& Musch, J. (2003). Affective priming: Findings and theories. In J. Musch \& K. Klauer (Eds.), The psychology of evaluation: Affective processes in cognition and emotion (pp. 7-49). Mahwah, NJ: Erlbaum.

Maxfield, L. (1997). Attention and semantic priming: A review of prime task effects. Consciousness and Cognition, 6, 204218.

Neely, J.H. (1977). Semantic priming and retrieval from lexical memory: Roles of inhibitionless spreading of activation and limited-capacity attention. Journal of Experimental Psychology: General, 106, 226-254.

Posner, M.I. , \& Snider, C.R.R. (1975). Facilitation and inhibition in the processing of signals. In P.N. Rabbit \& S.Dornic (Eds.), Attention and Performance. London: Academic Press.
Sollberger, B., Reber, R., \& Eckstein, D. (2003). Musical chords as affective priming context in a word-evaluation task. Music Perception, 20, 263-282.

Spruyt, A., Hermans, D., De Houwer, J., \& Eelen, P. (2002). On the nature of the affective priming effect: Affective priming of naming responses. Social Cognition, 20, 227-256.

Stenberg, G., Wiking, S. \& Dahl, M. (1998). Judging words at face value: Interference in a word processing task reveals automatic processing of affective facial expressions. Cognition and Emotion, 12, 755-782.

Stolz, J.A., \& Besner, D. (1999). On the myth of automatic semantic activation in reading. Current Directions in Psychological Science, 8, 61-65.

Received January 17, 2007

Revision received April 27, 2007

Accepted May 23, 2007 


\section{Appendix}

\section{Instructions of Experiment 1}

- In the following experiment you will see pictures of faces for a very short time. Sometimes, the face will be followed by a question mark and sometimes it will be followed by a word.

- When the question mark appears, you will have to indicate whether the face belongs to a male or to a female. When a word appears, you will have to decide whether you find it pleasant or unpleasant. For example, "toy" and "flower" are both pleasant, but "poison" and "punishment" are unpleasant.

- Remember: First you will see a face and then a question mark or a word.

- In the response box, press "1" for "male" and "2" for "female". Press these keys after the question mark.

- In the response box, press "4" for "pleasant" and "5" for "unpleasant". Press these keys when a word appears.

- An asterisk will appear on the center of the screen before each face. Remember that it is VERY IMPORTANT that you fix your eyes on the asterisk and keep your eyes fixed on the following image.

- Before the experiment starts, you will practice with a few examples. Don't forget to respond when the question mark or the word appears. 